\title{
Spatial Simon effects with nonspatial responses
}

\author{
JAN DE HOUWER \\ Ghent University, Ghent, Belgium
}

\begin{abstract}
Recent studies have shown that spatial Simon effects can be modulated by short-term associations that are set up as a result of task instructions. I examined whether spatial Simon effects can also be produced by short-term associations even when the responses are unrelated to spatial position. Participants were to say "cale" or "cole" on the basis of the direction of arrows (i.e., left or right), the meaning of words (i.e., left or right), and the color of squares presented left or right of the screen center. Responses to squares were faster when the correct response was associated with the same position as the irrelevantposition of the square (e.g., say "cale" to a square on the left when "cale" was assigned to the word left and the left arrow). This new type of stimulus-response compatibility effect provides the first evidence for short-term associations that involve mode-independent representations.
\end{abstract}

Stimulus-response compatibility (SRC) research has established that task performance is superior when the presented stimulus is somehow similar to the required response than when the stimulus and the response are dissimilar. As Proctor and Vu (2002) recently pointed out, most researchers subscribe to the idea that SRC effects are due to the existence of long-term memory associations between the representations of certain stimuli and the representations of certain responses. By long-term associations, they mean associations that are genetically determined or have been overlearned during the lifetime of an individual. For instance, in dual-route models, such as the highly influential model of Kornblum, Hasbroucq, and Osman (1990; see also De Jong, Liang, \& Lauber, 1994; Zorzi \& Umiltà, 1995), SRC effects are assumed to be the result of activation that automatically spreads from the stimulus representation to the response representation because of long-term associations between the two. When the automatically activated response representation is the representation of the required response, responses will be fast and accurate. When spreading of activation results in the activation of the incorrect response representation, this will hinder the selection of the correct response. Kornblum et al. (1990) emphasized that long-term stimulus-response associations exist only-and, thus, automatic response activation occurs only-when the set of stimuli and the set of responses have dimensions in common, or as they call it, when the stimulus and the response sets overlap.

Recent research, however, has clearly demonstrated that short-term associations between stimulus and response representations also have an important impact on SRC effects. Let us take the example of the well-known spatial Simon effect (see Lu \& Proctor, 1995, and Simon, 1990,

I thank Tom Randell for his help with collecting the data and Robert Proctor for pointing out relevant literature. Correspondence concerning this article should be addressed to J. De Houwer, Department of Psychology, Ghent University, Henri Dunantlaan 2, B-9000 Ghent, Belgium (e-mail: jan.dehouwer@ugent.be). for reviews). In a spatial Simon task, participants are asked to give lateralized responses on the basis of a nonspatial feature of stimuli that are presented in different locations. For instance, participants might be instructed to press a left key when they see a green circle and to press a right key when they see a blue circle. Importantly, the green and the blue circles are presented on the left or the right side of the screen. Results typically have shown that participants are faster to press the left key in response to a stimulus on the left side than to a stimulus on the right side, whereas the reverse is true when participants must press the right key. Most models account for the Simon effect by assuming that long-term associations exist between the representations of stimulus position (left or right) and the representations of response location (left or right) and that, as a result, a perceived stimulus automatically activates the response that has a corresponding location.

Proctor and Vu (2002) recently summarized an impressive series of studies that have demonstrated that the size and even the direction of the Simon effect can be modulated by short-term associations that are set up as the result of task instructions. Proctor and colleagues conducted studies in which they intermixed locationirrelevant and location-relevant trials. On each trial, they presented a circle either on the left or on the right side of the screen. On location-irrelevant trials, the participants were instructed to press a left or a right key according to the color of the circles while ignoring their position. On location-relevant trials, the participants also had to choose between the left and the right keypress response, but now on the basis of the location of the stimulus. The results consistently showed that performance on the locationirrelevant trials was influenced by whether the stimulusresponse mapping on the location-relevant trials was compatible or incompatible. When it was compatible (i.e., press the left key for left stimuli and press the right key for right stimuli), a strong Simon effect emerged on the location-irrelevant trials (i.e., better performance when the location of the correct response and the irrelevant location of the stimulus matched than when they dif- 
fered). However, when the stimulus-response mapping on the location-relevant trials was incompatible (i.e., press the left key for right stimuli and the right key for left stimuli), a reversed Simon effect was found: Performance was better when the position of the correct response and the location of the stimulus did not match (e.g., press left in response to the color of a stimulus presented on the right side) than when both positions matched (e.g., press left in response to the color of a left stimulus). A similar modulation of spatial Simon effects was found when the location-relevant trials were presented before, rather than intermixed with, the locationirrelevant trials (e.g., Proctor \& Lu, 1999; Tagliabue, Zorzi, Umiltà, \& Bassignani, 2000).

The results of all these studies suggest that short-term associations that are set up as the result of task instructions can have a major impact on performance in SRC tasks. Until now, however, researchers have investigated only whether Simon effects can be modulated by short-term associations between representations that are also linked by long-term associations (i.e., the representations of left and right stimulus and response location). The aim of the present study was to examine whether short-term associations also would influence performance when those associations link representations that are not linked by long-term associations. That is, I examined whether short-term associations can in and of themselves produce a Simon effect.

The present experiment consisted of location-relevant and location-irrelevant trials. On location-relevant trials, the participants saw the word left, the word right, an arrow pointing to the left, or an arrow pointing to the right. Half of the participants were instructed to say "cale" in response to the word left and the left arrow and to say "cole" when the word right or the right arrow was presented. The other participants received the opposite instructions (i.e., say "cale" to right and the right arrow; say "cole" to left and the left arrow). One can assume that as a result of these instructions, short-term associations are set up between the representations of the arbitrary responses "cale" and "cole," on the one hand, and the representations of the concepts left and right, on the other hand. Importantly, there are no reasons to assume that these representations are linked by long-term associations. On the location-irrelevant trials, blue and green squares were presented on the left or the right side of the screen, and the participants were instructed to say "cale" or "cole" on the basis of the color of the square while ignoring its position. If short-term associations can support SRC effects, responses to the squares should be faster when the correct response is associated with the same position as the irrelevant position of the square.

\section{METHOD}

\section{Participants}

Forty-four psychology undergraduates at the University of Southampton participated for partial fulfillment of course requirements. All had normal or corrected-to-normal vision.

\section{Stimuli and Apparatus}

All the stimuli were presented on a 14 -in. screen that was connected to an IBM-compatible 486 computer. The blue and green squares were $9 \mathrm{~mm}$ high and wide, the words left and right were presented in white uppercase letters that were $7 \mathrm{~mm}$ high and $6 \mathrm{~mm}$ wide, and the arrows were also presented in white and were $7 \mathrm{~mm}$ high and $2 \mathrm{~cm}$ wide. The words and arrows were presented in the middle of the screen. The squares were presented $1.5 \mathrm{~cm}$ to the left or the right of the screen center. A voice key that was connected to the game port of the computer registered the verbal responses. Presentation of the stimuli and registration of the responses was controlled by a custom-made Turbo Pascal 5.0 program that included a highly accurate timer (Bovens \& Brysbaert, 1990).

\section{Procedure}

The participants were tested individually in a dimly lit room and were seated approximately $50 \mathrm{~cm}$ from the computer screen. Instructions that appeared on the screen informed the participants that words, arrows, and colored squares would be presented one by one. Their task was to say "cale" or "cole," depending on the meaning of the word, the direction of the arrow, or the color of the square. Then they saw the particular stimulus-response assignments. For half of the participants, "cale" was assigned to the word left and the left arrow, and "cole" was assigned to the word right and the right arrow. The reverse was true for the other participants. Orthogonal to this manipulation, half of the participants were instructed to say "cale" in response to blue squares and "cole" to green squares, whereas the color-response assignments were reversed for the other the participants. Finally, participants were informed that incorrect responses would be indicated by a short beep and that the experiment would consist of three series of practice trials and four series of test trials.

All the participants first completed a practice block in which only words and arrows were presented. Each word and arrow appeared on 5 trials, resulting in a total of 20 trials. During the second practice block of 20 trials, the blue and green squares were each presented 5 times on the left side of the screen and 5 times on the right side. The third practice block also consisted of 20 trials. These 20 trials were the first of a series of 24 trials during which each word and arrow was presented 3 times and each colored square was presented 3 times on the left and 3 times on the right. Next, two blocks of 60 experimental trials were presented. In each series, the two words and arrows each appeared 5 times, whereas the green and blue squares were presented 10 times on the left side and 10 times on the right side. The order of the trials within each practice and experimental block was randomized, with the restriction that the stimulus (word, arrow, blue square, or green square) could not be the same on more than 2 consecutive trials and that the correct response could not be the same on 4 or more consecutive trials. The participants could take a break after reading the instructions, after each practice block, and after each series of 30 experimental trials. During this break, instructions appeared on the screen that informed the participants about whether the next series would be for practice or test and about the nature of the stimuli that would appear during that series of trials (i.e., words and arrows only, squares only, or all the stimuli). The participants could end the break by pressing the Enter key. A warm-up trial immediately preceded each of the four series of 30 experimental trials. These 4 warm-up trials were the remaining trials from the series of 24 trials from which the 20 trials of the third practice block were selected.

Each practice, warm-up, and experimental trial started with the presentation of a fixation cross for $500 \mathrm{msec}$, immediately followed by a stimulus that stayed on the screen until the voice key registered a response or 3,000 msec elapsed. The experimenter then coded the response of the participant or entered a separate code if the voice key did not accurately register the verbal response. If the response was incorrect, a $200-\mathrm{Hz}$ tone was presented for $250 \mathrm{msec}$. The next 
trial started 1,500 msec after the experimenter entered the code or after the end of the feedback tone.

\section{RESULTS}

I first analyzed reaction times and response accuracy on the experimental trials in which a colored square was presented. Reaction times shorter than $150 \mathrm{msec}$ or longer than $1,500 \mathrm{msec}$ were excluded $(0.63 \%$ of all trials), as were reaction times on trials in which the voice key did not accurately register the response $(2.81 \%$ of all the trials) or an incorrect response was given $(2.86 \%$ of the remaining trials). One participant had a mean reaction time that was more than three standard deviations higher than the mean reaction time for the total group and also made about seven times more errors than the average number of errors for the total group. Her data were therefore excluded, leaving the data of 43 participants for analysis. For each block of 60 trials, I calculated the mean reaction time and percentage of errors on congruent and incongruent trials. Congruent trials were trials on which the position of the square corresponded to the location with which the response was associated. Incongruent trials were trials on which these positions differed. For instance, if the word left and the left arrow were assigned to the response "cale," a congruent trial was a trial on which "cale" was the correct response and the square was presented on the left side, whereas the trial would be incongruent if the same square was presented on the right side.

I also analyzed the data of the second practice block, in which only colored squares were presented. Given that this practice block was preceded by a practice block in which only words and squares were presented, one can assume that the responses were already associated with the concepts left and right as a result of the first practice block. If these short-term associations remain active even during a task in which they are not relevant anymore (i.e., in which only squares are presented), one could expect that performance during the second practice block would be influenced by whether the correct response had been previously assigned to the same position as the position on which the square was presented. I therefore calculated the mean reaction time and percentage of errors on congruent and incongruent trials of the second practice phase, using the same method of data reduction as that for the analysis of the experimental trials. The verbal response was not accurately registered on $10.7 \%$ of the relevant practice trials, an incorrect response was given on $10.08 \%$ of the remaining trials, and $1.40 \%$ of the reaction times were shorter than $150 \mathrm{msec}$ or longer than $1,500 \mathrm{msec}$. The participant whose data were excluded from the analyses of experimental trials was also more than three standard deviations slower and less accurate on the practice trials than the remaining participants. Therefore, her data were also excluded from these analyses.
A block $\times$ congruence analysis of variance (ANOVA) on the reaction time data of the experimental trials revealed a significant main effect of congruence $[F(1,42)=$ $14.25, p<.001]$. Table 1 shows that reaction times were faster on congruent than on incongruent trials. The main effect of block was also significant $[F(1,42)=10.08$, $p=.003]$, due to faster responses in the second than in the first block. The interaction between both variables was not significant $(F<1)$. A similar analysis of the percentage of errors revealed only a main effect of block $[F(1,42)=18.55, p<.001]$, which indicated that fewer errors were made in the second than in the first block $\left(F_{\mathrm{S}}<1\right.$ for all other effects).

An ANOVA with congruence as the only withinsubjects variable was conducted on the reaction time data of the second practice block. The main effect was significant $[F(1,42)=7.28, p=.01]$, showing that responses were faster on congruent $(M=585, S D=162)$ than on incongruent $(M=635, S D=173)$ practice trials. The ANOVA on the error data of the practice trials did not reveal a main effect of congruence $(F<1)$.

\section{DISCUSSION}

The present results provide the first demonstration of a spatial Simon effect in which stimuli and responses are related only as the result of short-term associations. Such an effect occurred both when location-relevant trials (i.e., trials with words or arrows) were intermixed with location-irrelevant trials (i.e., trials with colored squares) and when participants knew that only location-irrelevant trials would be presented. The latter result suggests that the short-term associations that are set up during one task (i.e., the practice block with only words and arrows) remain active even when those associations are no longer task relevant. The present findings are in line with the results of previous studies that have shown that the compatibility of a location-relevant task influences performance on location-irrelevant trials both when the locationrelevant trials precede the location-irrelevant trials (e.g., Proctor \& Lu, 1999; Tagliabue et al., 2000) and when location-relevant and location-irrelevant trials are intermixed (see Proctor \& Vu, 2002, for a review). It is also interesting to note that I have replicated the present results by using stimulus valence (i.e., good or bad), rather than location, as the crucial stimulus dimension (De Houwer, 2003). This attests to the generality and reliability of the

Table 1

Mean Reaction Times (RTs) and Percentages of Errors (PEs) as a Function of Block and Congruence

\begin{tabular}{|c|c|c|c|c|c|c|c|c|}
\hline \multirow[b]{3}{*}{ Congruence } & \multicolumn{4}{|c|}{ Block 1} & \multicolumn{4}{|c|}{ Block 2} \\
\hline & \multicolumn{2}{|c|}{ RT } & \multicolumn{2}{|c|}{$\mathrm{PE}$} & \multicolumn{2}{|c|}{ RT } & \multicolumn{2}{|c|}{$\mathrm{PE}$} \\
\hline & $M$ & $S D$ & $M$ & $S D$ & $M$ & $S D$ & $M$ & $S D$ \\
\hline Congruent & 618 & 137 & 4.23 & 6.74 & 592 & 136 & 0.98 & 2.35 \\
\hline Incongruent & 651 & 160 & 3.44 & 5.41 & 624 & 137 & 1.44 & 3.62 \\
\hline
\end{tabular}


new form of Simon effect that was introduced in this article.

This new form of Simon effect extends previous findings not only by demonstrating the impact of short-term associations in the absence of long-term associations, but also by providing the first indication that short-term associations are not necessarily specific to the mode (e.g., verbal, symbolic, or physical location) in which location information is conveyed. Proctor, Marble, and $\mathrm{Vu}$ (2000) found that Simon effects were modulated by the compatibility of the location-relevant task only when the stimuli on the location-relevant and the location-irrelevant trials were from the same mode (e.g., all words, all arrows, or all circles presented on the left or the right), but not when they were from different modes (e.g., circles on the left or the right and the words left or right). ${ }^{1}$ On the basis of these and other results, they concluded that short-term associations involve mode-specific stimulus representations (as is assumed in the model of Cohen, Dunbar, \& McClelland, 1990), rather than abstract semantic stimulus representations (as is assumed in the model of Zhang \& Kornblum, 1998). In the present study, however, location information was conveyed in a different mode on the location-relevant trials (verbal and symbolic) than on the location-irrelevant trials (physical location). The fact that a Simon effect was found suggests that the crucial short-term associations did involve mode-independent stimulus representations.

A crucial difference between the present experiment and those of Proctor et al. (2000) is that in the former, two kinds of stimuli were presented on location-relevant trials (words and arrows), whereas in the latter, only one type of stimulus was presented on those trials (e.g., only left or right circles). When both the word left and a left arrow are mapped onto the same response (e.g., say "cale") and the word right and a right arrow are assigned to a second response (e.g., say "cole"), it is indeed more efficient to create two short-term associations that involve the abstract representations of left and right than to create four mode-specific short-term associations for each type of stimulus. It would, thus, be interesting to replicate the studies of Proctor et al. (2000), but now with location-relevant stimuli of more than one stimulus mode (e.g., verbal and symbolic). If the compatibility of the location-relevant task does modulate spatial Simon effects under these conditions, it would support the hypothesis that short-term associations involve modeindependent stimulus representations when more than one stimulus mode is relevant on location-relevant trials. Likewise, it is possible that the Simon effect that was observed in the present study would not have been found if only words or only arrows had been presented on the location-relevant trials. Such additional studies could provide important information about the way in which short-term associations are set up. But regardless of the outcome of those studies, the present results demonstrate that short-term associations are not always mode specific.
Results such as those reported in this article have important implications for models of SRC performance. As was noted in the introduction, dual-route models postulate that irrelevant stimulus information can influence performance only as the result of activation that spreads through long-term associations (e.g., De Jong et al., 1994; Kornblum et al., 1990; Zorzi \& Umiltà, 1995). The present study provides particularly strong support for the hypothesis that activation caused by the presentation of irrelevant stimulus information can also spread through short-term associations that are set up as the result of task instructions. In previous studies on the role of shortterm associations, stimuli and responses were linked by both short-term and long-term associations. Thus, one could argue that the observed effects of location-relevant trials were due to a modulation of the amount of activation that spread through long-term associations, rather than to activation that spread through short-term associations. Although there are good arguments against such an alternative account (see Tagliabue et al., 2000), it is even less probable for the effects that were observed in the present study, simply because stimuli and responses were not linked by long-term associations.

One might argue that the location-relevant task in the present study did not result in the creation of short-term associations but led to the development of long-term associations. This, of course, raises the question of how long- and short-term associations differ. It is generally assumed that the crucial difference lies in the fact that long-term associations develop slowly as the result of practice, whereas short-term associations are set up quickly as the result of task instructions (e.g., Barber \& O'Leary, 1997; McClelland, McNaughton, \& O'Reilly, 1995; Tagliabue et al., 2000). In the present study, a Simon effect was found on location-irrelevant practice trials that were preceded by only 20 location-relevant trials. Moreover, when location-relevant and location-irrelevant trials were intermixed, the Simon effect did not increase over blocks. Both findings suggest that the observed Simon effect was based primarily on the operation of short-term associations that were set up as the result of task instructions (see also Tagliabue et al., 2000). ${ }^{2}$ It would be interesting to examine whether the present results can be replicated when participants are given instructions on how to respond to location-relevant and location-irrelevant trials but are not actually given location-relevant trials. If a Simon effect is found on the location-irrelevant trials, this would provide even stronger evidence against an account in terms of long-term associations. Nevertheless, the present results clearly strengthen the conclusion that dual-route models need to be modified in such a way that activation is allowed to spread automatically through short-term associations (see Tagliabue et al., 2000, and Zhang \& Kornblum, 1998, for examples of such models). In fact, all future models of SRC performance should be able to accommodate the pervasive effects of short-term associations on performance in SRC tasks. 


\section{REFERENCES}

BARBER,P., \& O'LEARY, M. (1997). The relevance of salience: Towards an activational account of irrelevant stimulus-response compatibility effects. In B. Hommel \& W. Prinz (Eds.), Theoretical issues in stimulusresponse compatibility (pp. 135-172). Amsterdam: North-Holland.

Bovens, N., \& BRYSBAERT, M. (1990). IBM PC/XT/AT and PS/2 Turbo Pascal timing with extended resolution. Behavior Research Methods, Instruments, \& Computers, 22, 332-334.

Cohen, J. D., Dunbar, K., \& MCClelland, J. L. (1990). On the control of automatic processes: A parallel distributed processing account of the Stroop effect. Psychological Review, 97, 332-361.

De Houwer, J. (2003). The extrinsic affective Simon task. Experimental Psychology, 50, 77-85.

De Jong, R., Liang, C. C., \& LAuber, E. (1994). Conditional and unconditional automaticity: A dual-process model of effects of spatial stimulus-response correspondence. Journal of Experimental Psychology: Human Perception \& Performance, 20, 731-750.

Homme L, B. (1996). The cognitive representation of action: Automatic integration of perceived action effects. Psychological Research, 59, 176-186.

Hommel, B. (1997). Toward an action-concept model of stimulusresponse compatibility. In B. Hommel \& W. Prinz (Eds.), Theoretical issues in stimulus-response compatibility (pp. 281-320). Amsterdam: North-Holland.

Kornblum, S., HasbroucQ, T., \& Osman, A. (1990). Dimensional overlap: Cognitive basis for stimulus-response compatibility: A model and taxonomy. Psychological Review, 97, 253-270.

LIPPA, Y., \& GOLDSTONE, R. L. (2001). The acquisition of automatic response biases through categorization. Memory \& Cognition, 29, 1051-1060.

Lu, C.-H., \& Proctor, R. W. (1995). The influence of irrelevant location information on performance: A review of the Simon and spatial Stroop effects. Psychonomic Bulletin \& Review, 2, 174-207.

MCClelland, J. L., MCNaughton, B. L., \& O'Reilly, R. C. (1995). Why there are complementary learning systems in the hippocampus and neocortex: Insights from the successes and failures of connectionist models of learning and memory. Psychological Review, 102, 419-437.

Proctor, R. W., \& LU, C.-H. (1999). Processing irrelevant location information: Practice and transfer effects in choice-reaction tasks. Memory \& Cognition, 27, 63-77.

Proctor, R. W., Marble, J. G., \& VU, K.-P. L. (2000). Mixing incompatible mapped location-relevant trials with location-irrelevant trials: Effects of stimulus mode on the reverse Simon effect. Psychological Research, 64, 11-24.

Proctor, R. W., \& VU, K.-P. L. (2002). Eliminating, magnifying, and reversing spatial compatibility effects with mixed location-relevant and irrelevant trials. In W. Prinz \& B. Hommel (Eds.), Common mechanisms in perception and action: Attention and performance XIX (pp. 443-473). Oxford: Oxford University Press.

SimON, J. R. (1990). The effects of an irrelevant directional cue on human information processing. In R. W. Proctor \& T. G. Reeve (Eds.),
Stimulus-response compatibility: An integrated perspective (pp. 3186). Amsterdam: North-Holland.

Tagliabue, M., Zorzi, M., \& Umiltà, C. (2002). Cross-modal remapping influences the Simon effect. Memory \& Cognition, 30, 18-23.

Tagliabue, M., Zorzi, M., Umiltà, C., \& Bassignani, F. (2000). The role of long-term-memory and short-term-memory links in the Simon effect. Journal of Experimental Psychology: Human Perception \& Performance, 26, 648-670.

Toth, J. P., Levine, B., Stuss, D. T., OH, A., Winocur, G., \& Meiran, N. (1995). Dissociation of processes underlying S-R compatibility: Evidence for the independent influence of what and where. Consciousness \& Cognition, 4, 483-501.

ZHANG, H., \& KoRNBLUM, S. (1998). The effects of stimulus-response mapping and irrelevant stimulus-response and stimulus-stimulus overlap in four-choice Stroop tasks with single-carrier stimuli. Journal of Experimental Psychology: Human Perception \& Performance, 24, 3-19.

ZorZI, M., \& UMILTÀ, C. (1995). A computational model of the Simon effect. Psychological Research, 58, 193-205.

\section{NOTES}

1. Tagliabue, Zorzi, and Umiltà (2002) recently demonstrated that the compatibility of a location-relevant task influenced performance on a location-irrelevant task even when the stimuli in both tasks were of a different sensory modality (i.e., auditory or visual stimuli, presented on the left or the right). This suggests that short-term associations are not necessarily specific to a sensory modality. As Tagliabue et al. (2002) noted, their findings do not imply that short-term associations are independent of the mode in which the location information is presented, because, in their experiments, location information was always conveyed through physical position.

2. This does not exclude the possibility that Simon effects can result from or be modulated by associative influences other than those of short-term stimulus-response associations that are induced by task instructions. First, practice can result in long-term associations between responses and effects of those responses. For instance, Hommel (1996) showed that, after substantial practice, nonspatial responses can become associated with spatial action effects and, as a result, can be emitted more quickly in response to stimuli that are spatially compatible with the associated action effect. Note that in terms of Hommel's (1997)actionconcept model, the present results imply that action concepts are determined not only by the consequences of actions, but also by their antecedents or determinants. Second, spatially neutral stimuli can be associated with spatially relevant stimuli and, as a result, activate spatial responses (e.g., Lippa \& Goldstone, 2001). Third, there is some evidence that stimulus-response associations can also be influenced by variations in the contingency between irrelevant stimulus features and relevant response features, variations that result from differences in the proportion of incongruent trials (e.g., Toth et al., 1995).

(Manuscript received June 14, 2002; revision accepted for publication September 5, 2002.) 